B. BECKERMANN (Lille and Toulon)

J. GILEWICZ and E. LEOPOLD (Toulon)

\title{
RECURRENCE RELATIONS \\ WITH PERIODIC COEFFICIENTS \\ AND CHEBYSHEV POLYNOMIALS
}

Abstract. We show that polynomials defined by recurrence relations with periodic coefficients may be represented with the help of Chebyshev polynomials of the second kind.

Introduction. The class of orthogonal polynomials studied in this paper served as a starting point for several authors [1-4] when studying more general classes of orthogonal polynomials or continued fractions. The aim of this note is to show that, on the other hand, this class can be described with the help of the classical Chebyshev polynomials.

Let $\left\{P_{n}\right\}_{n}, \operatorname{deg} P_{n}=n$, be a sequence of polynomials defined by a threeterm recurrence

$$
\begin{gathered}
\forall n>0: \quad P_{n}(z)=\left(z+b_{n-1}\right) P_{n-1}(z)-a_{n-1} P_{n-2}(z), \\
P_{-1} \equiv 0, \quad P_{0} \equiv 1,
\end{gathered}
$$

where the coefficients are periodic with period $p$ :

$$
\forall n \geq 0: \quad a_{n+p}=a_{n}, \quad b_{n+p}=b_{n} .
$$

Note that for convenience we define $a_{0}:=a_{p}$. The case $p=1$ immediately leads to the classical Chebyshev polynomials. We will show that, for arbitrary $p$, the polynomials $P_{n}$ may also be expressed with the help of the Chebyshev polynomials of the second kind. For the special case $p=2$ and $b_{n}=0$, such a representation has been given in $[1$, p. 91]. The case of asymptotically periodic recurrence coefficients was studied in [2].

1991 Mathematics Subject Classification: 12D10, 33C25.

Key words and phrases: orthogonal polynomials, periodic coefficients of recurrence relation. 
The results of the first section do not require any additional assumptions, hence they remain valid also in the case of complex coefficients. In contrast, for our main result of Section II we will restrict ourselves to the case of real coefficients.

I. Some properties induced by the periodicity. In the considerations to follow we will often omit the argument and write simply $P_{n}$ instead of $P_{n}(z)$.

Property 1. The polynomial $P_{p-1}$ divides the polynomial $P_{2 p-1}$ :

$$
\forall p>0: \quad P_{2 p-1}(z)=Q_{p}(z) P_{p-1}(z),
$$

where

$$
\begin{aligned}
& Q_{1}(z)=P_{1}(z), \\
& Q_{2}(z)=P_{2}(z)-a_{0} \\
& Q_{p}(z)=P_{p}(z)-a_{0}\left[\prod_{i=1}^{p-2}\left(z+b_{i}\right)+R_{p-4}(z)\right], \quad p \geq 3,
\end{aligned}
$$

and $R_{k}$ is a polynomial of degree $k\left(R_{-1} \equiv 0\right)$.

Proof. The case $p=1$ is trivial. For $p \geq 2$, using (1) and (2) we obtain

$$
\begin{aligned}
\left|\begin{array}{cc}
P_{2 p-1} & P_{p} \\
P_{p-1} & P_{0}
\end{array}\right| & =\left|\begin{array}{ll}
\left(z+b_{2 p-2}\right) P_{2 p-2}-a_{2 p-2} P_{2 p-3} & P_{p} \\
\left(z+b_{p-2}\right) P_{p-2}-a_{p-2} P_{p-3} & P_{0}
\end{array}\right| \\
& =\left(z+b_{p-2}\right)\left|\begin{array}{cc}
P_{2 p-2} & P_{p} \\
P_{p-2} & P_{0}
\end{array}\right|-a_{p-2}\left|\begin{array}{cc}
P_{2 p-3} & P_{p} \\
P_{p-3} & P_{0}
\end{array}\right| .
\end{aligned}
$$

In the case $p=2$, the first term vanishes, and the assertion follows immediately:

$$
\left|\begin{array}{cc}
P_{3} & P_{2} \\
P_{1} & 1
\end{array}\right|=-a_{0}\left|\begin{array}{ll}
P_{1} & P_{2} \\
0 & 1
\end{array}\right|, \quad \text { that is, } P_{3}=\left(P_{2}-a_{0}\right) P_{1} .
$$

If $p \geq 3$, we apply the recurrence relation (1) for $P_{2 p-2}$ and $P_{p-2}$, so that together with the periodicity $(2)$ the above expression can be rewritten as $\left[\left(z+b_{p-2}\right)\left(z+b_{p-3}\right)-a_{p-2}\right]\left|\begin{array}{cc}P_{2 p-3} & P_{p} \\ P_{p-3} & P_{0}\end{array}\right|-a_{p-3}\left(z+b_{p-2}\right)\left|\begin{array}{cc}P_{2 p-4} & P_{p} \\ P_{p-4} & P_{0}\end{array}\right|$.

As before, for $p=3$ the left hand term vanishes yielding the assertion, whereas for $p>3$ we continue to transform the left hand term by applying the recurrence relation (1) for $P_{2 p-3}$ and $P_{p-3}$. The assertion (3) for arbitrary $p$ follows after $p-1$ such transformations.

Later we shall obtain a more general result than (3) (formula (14)): 
Property 1 (generalized). The polynomial $P_{p-1}$ divides the polynomial $P_{k p-1}$ for any positive integer $k$.

Property 2.

$$
\forall n \geq-1: \quad\left|\begin{array}{ll}
P_{2 p+n}(z) & P_{2 p-1}(z) \\
P_{p+n}(z) & P_{p-1}(z)
\end{array}\right|=-a P_{p-1}(z) P_{n}(z),
$$

where $a=\prod_{i=0}^{p-1} a_{i}$.

Proof. Property 2 will be shown by induction on $n \geq-1$, the case $n=-1$ is trivial. For $n=0$, we get with the help of (1) and (2),

$$
\begin{aligned}
\left|\begin{array}{cc}
P_{2 p} & P_{2 p-1} \\
P_{p} & P_{p-1}
\end{array}\right| & =\left|\begin{array}{cc}
\left(z+b_{p-1}\right) P_{2 p-1} & P_{2 p-1} \\
\left(z+b_{p-1}\right) P_{p-1} & P_{p-1}
\end{array}\right|+a_{p-1}\left|\begin{array}{cc}
P_{2 p-1} & P_{2 p-2} \\
P_{p-1} & P_{p-2}
\end{array}\right| \\
& =\ldots=\prod_{i=0}^{p-1} a_{i}\left|\begin{array}{cc}
P_{p} & P_{p-1} \\
P_{0} & P_{-1}
\end{array}\right| \equiv-a P_{p-1} .
\end{aligned}
$$

Supposing now that assertion (5) holds for $n \leq k$, one obtains for $n=k+1$ :

$$
\begin{aligned}
\left|\begin{array}{cc}
P_{2 p+k+1} & P_{2 p-1} \\
P_{p+k+1} & P_{p-1}
\end{array}\right| & =\left(z+b_{k}\right)\left|\begin{array}{cc}
P_{2 p+k} & P_{2 p-1} \\
P_{p+k} & P_{p-1}
\end{array}\right|-a_{k}\left|\begin{array}{cc}
P_{2 p+k-1} & P_{2 p-1} \\
P_{p+k} & P_{p-1}
\end{array}\right| \\
& =-a\left(z+b_{k}\right) P_{p-1} P_{k}+a a_{k} P_{p-1} P_{k-1}=-a P_{p-1} P_{k+1} .
\end{aligned}
$$

PROPERTY 3.

(6)

$$
\forall n \geq-1: \quad P_{2 p+n}(z)=Q_{p}(z) P_{p+n}(z)-a P_{n}(z) .
$$

This is clearly formula (5) modified by using (4). The similar result was already derived in [2] and [3].

Property 4. Let the sequence of polynomials $\left\{V_{n}\right\}_{n}$ be defined by

$$
\forall n \geq 0: \quad V_{n+1}(z)=z V_{n}(z)-a V_{n-1}(z), \quad V_{-1} \equiv 0, V_{0} \equiv 1 .
$$

Then

(8) $\forall n \geq-1, \forall k \geq 1$ :

$$
P_{k p+n}(z)=P_{p+n}(z) V_{k-1}\left[Q_{p}(z)\right]-a P_{n}(z) V_{k-2}\left[Q_{p}(z)\right] .
$$

Proof. The assertion will be shown by induction on $k \geq 1$. The case $k=1$ is trivial, and for $k=2$ equation (8) coincides with (6). Suppose the result is true for $k$ and let us verify it for $k+1$. Replacing $n$ by $n+p$ in (8) yields

$$
\begin{aligned}
P_{(k+1) p+n}(z) & =P_{k p+(n+p)}(z) \\
& =P_{2 p+n}(z) V_{k-1}\left[Q_{p}(z)\right]-a P_{n+p}(z) V_{k-2}\left[Q_{p}(z)\right] .
\end{aligned}
$$

By (6) this equals 


$$
\begin{aligned}
& {\left[Q_{p}(z) P_{p+n}(z)-a P_{n}(z)\right] V_{k-1}\left[Q_{p}(z)\right]-a P_{p+n}(z) V_{k-2}\left[Q_{p}(z)\right]} \\
& \quad=P_{p+n}(z)\left\{Q_{p}(z) V_{k-1}\left[Q_{p}(z)\right]-a V_{k-2}\left[Q_{p}(z)\right]\right\}-a P_{n}(z) V_{k-1}\left[Q_{p}(z)\right] \\
& \quad=P_{p+n}(z) V_{k}\left[Q_{p}(z)\right]-a P_{n}(z) V_{k-1}\left[Q_{p}(z)\right]
\end{aligned}
$$

leading to the result for $k+1$.

II. A connection between the polynomials $P_{n}$ and the Chebyshev polynomials of the second kind $U_{n}$. In the sequel, the periodic coefficients $a_{n}$ are supposed to be positive, and $b_{n}$ real:

$$
\exists p \geq 1, \forall n \geq 0: \quad a_{n+p}=a_{n}>0, \quad b_{n+p}=b_{n} \in \mathbb{R} .
$$

The Chebyshev polynomials of the second kind $U_{n}$ are defined by the recurrence relation

$$
\forall n \geq 0: \quad U_{n+1}(x)=2 x U_{n}(x)-U_{n-1}(x), \quad U_{-1} \equiv 0, U_{0} \equiv 1,
$$

or, explicitly, by

$$
U_{n}(x)=\frac{\sin [(n+1) \arccos (x)]}{\sin [\arccos (x)]}, \quad x \in[-1,+1] .
$$

In fact, the polynomials $V_{n}$ introduced in (7) coincide, up to a simple transformation, with the Chebyshev polynomials of the second kind:

$$
V_{n}(x)=(\sqrt{a})^{n} U_{n}\left(\frac{x}{2 \sqrt{a}}\right) .
$$

Therefore, equation (8) leads immediately to our main result:

THEOREM. The polynomials $P_{j}$ defined by the recurrence relation (1) with coefficients satisfying the conditions (9) can be expressed as follows:

(12) $\forall k \geq 1, \forall n \geq-1$ :

$$
P_{k p+n}(x)=(\sqrt{a})^{k-1}\left\{P_{p+n}(x) U_{k-1}\left[\frac{Q_{p}(x)}{2 \sqrt{a}}\right]-\sqrt{a} P_{n}(x) U_{k-2}\left[\frac{Q_{p}(x)}{2 \sqrt{a}}\right]\right\},
$$

where $Q_{p}(x)=P_{2 p-1}(x) / P_{p-1}(x)$ is defined by (4) and $a=\prod_{i=0}^{p-1} a_{i}$.

Remark 1. As we have already mentioned in the introduction, for the particular case $p=1$, the polynomials $P_{k}$ coincide, up to a simple transformation, with the Chebyshev polynomials. More precisely, from (12) with $n=0$ we conclude that

$$
\begin{aligned}
P_{k}(x) & =(\sqrt{a})^{k-1}\left\{P_{1}(x) U_{k-1}\left[\frac{P_{1}(x)}{2 \sqrt{a}}\right]-\sqrt{a} U_{k-2}\left[\frac{P_{1}(x)}{2 \sqrt{a}}\right]\right\} \\
& =(\sqrt{a})^{k}\left\{2 \frac{P_{1}(x)}{2 \sqrt{a}} U_{k-1}\left[\frac{P_{1}(x)}{2 \sqrt{a}}\right]-U_{k-2}\left[\frac{P_{1}(x)}{2 \sqrt{a}}\right]\right\},
\end{aligned}
$$


that is,

$$
P_{k}(x)=(\sqrt{a})^{k} U_{k}\left[\frac{P_{1}(x)}{2 \sqrt{a}}\right] .
$$

Rem ark 2. Taking $n=-1$ in (12) leads to

$$
P_{k p-1}(x)=(\sqrt{a})^{k-1} P_{p-1}(x) U_{k-1}\left[\frac{Q_{p}(x)}{2 \sqrt{a}}\right],
$$

generalizing Property 1.

Conclusion. In the proof given above we have not required the part of the condition (9) that the coefficients $b_{j}$ are assumed to be real. However, this assumption completes the conditions on the coefficients of a three-term recurrence (1) to generate a sequence of orthogonal polynomials. Finally, we notice that the result of our Theorem may simplify the study of zeros of the polynomials $P_{n}$ since the zeros of the Chebyshev polynomials are explicitly known.

\section{References}

[1] T. S. Chihara, An Introduction to Orthogonal Polynomials, Gordon and Breach, 1978.

[2] J. S. Geronimo and W. Van Assche, Orthogonal polynomials with asymptotically periodic recurrence coefficients, J. Approx. Theory 46 (1986), 251-283.

[3] -, -, Approximating the weight function for orthogonal polynomials on several intervals, ibid. 65 (1991), 341-371.

[4] H. S. Wall, Analytic Theory of Continued Fractions, D. Van Nostrand, 1967.

BERNHARD BECKERMANN

LABORATOIRE D'ANALYSE NUMÉRIQUE ET D'OPTIMISATION

UFR IEEA-M3

USTL FLANDRES-ARTOIS

59655 VILLENEUVE D'ASCQ CEDEX, FRANCE

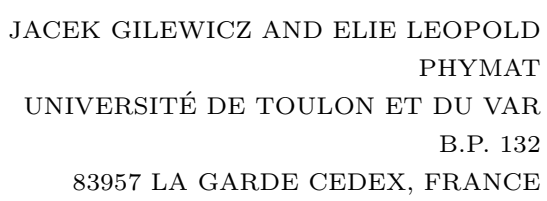

JACEK GILEWICZ AND ELIE LEOPOLD DU VAR 83957 LA GARDE CEDEX, FRANCE 